\title{
Characterization of ultrashort pulses by a modified grating-eliminated no-nonsense observation of ultrafast incident laser light $E$ fields (GRENOUILLE) method
}

\author{
Ismael A. Heisler, Ricardo R. B. Correia, and Silvio L. S. Cunha
}

\begin{abstract}
The measurement and characterization of ultrashort laser pulses remains an arduous task. The most commonly used pulse-measurement method is known as frequency-resolved optical gating (FROG), and another version with great experimental simplification and low-priced setup is known as gratingeliminated no-nonsense observation of ultrafast incident laser light $E$ fields (GRENOUILLE). Nevertheless, there is interest in elaborating other, more accessible or simpler and cheaper, setups with equal or better assets. We explored modification of the GRENOUILLE method in which we replaced the original Fresnel biprism with a beam splitter and two mirrors and used a cheap webcam to measure the pulse traces. We have evaluated our system, and we propose a method to correct border effects caused by the beam intensity's profile based on the characterization of three pulse classes: Fourier-transform limited, double, and chirped. We compare the recovered electric field with further spectral and second-order correlation data of the corresponding pulses. (C) 2005 Optical Society of America

OCIS codes: $320.0320,320.7100,190.7110$.
\end{abstract}

\section{Introduction}

Measuring ultrashort laser pulses has always been a challenge for physicists. For many years it was possible to create ultrashort pulses but not to characterize them completely. Precise knowledge of temporal pulse evolution is necessary for verifying theoretical models of pulse generation. Also, to make even shorter pulses it is indispensable to understand the distortions that limit the lengths of currently available pulses. In experiments in which such pulses are used it is always important to know at least the pulse duration to determine the temporal resolution of a given experiment. Finally, in many experiments (involving the steering of chemical reactions, for example), additional details of the pulse's structure can play an important role in determining the outcome of the experiment. The coherent control achieved by

The authors are with the Instituto de Física, Universidade Federal do Rio Grande do Sul, Avenida Bento Gonçalves, 9500, Campus do Vale, Caixa Postal 15051, CEP 91501-970, Porto Alegre, RS, Brazil. R. R. B. Correia's e-mail address is rego@if.ufrgs.br.

Received 9 June 2004; revised manuscript received 8 September 2004; accepted 11 September 2004.

0003-6935/05/163377-06\$15.00/0

(C) 2005 Optical Society of America shaping light pulses to have rather specific interactions with specific material systems has called attention to the technology of tailoring and measuring light fields. ${ }^{1-4}$ Fortunately, remarkable progress has occurred in the development of techniques for the measurement of ultrashort laser pulses. The most commonly used pulse-measurement methods (useful down to the few-cycle level) are frequency-resolved optical gating5,6 (FROG) and spectral phase interferometry for direct electric-field reconstruction 7,8 (SPIDER), both of which exist in numerous versions. These methods have achieved high accuracy ${ }^{9}$ and high precision in matching the reconstructed electric field. ${ }^{10} \mathrm{~A}$ well-known simplified variation of the FROG method is grating eliminated nonsense observation of ultrafast incident laser light $E$ fields ${ }^{11}$ (GRENOUILLE), in which a great amount of experimental simplification was introduced. In this setup the wave front is split and crossed by a Fresnel biprism, permitting single-shot pulse analysis.

We explore a modified version of GRENOUILLE, which constitutes an alternative for those who do not have a matching Fresnel biprism on the shelf but who are interested in a similar simple setup. As we show, this modified version adds some new features that were not contemplated in the biprism original GRENOUILLE setup with a fixed apex angle. The 


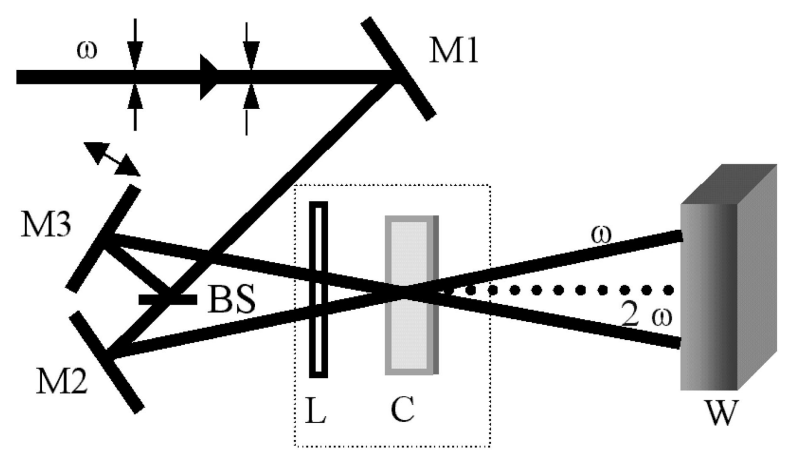

(a)

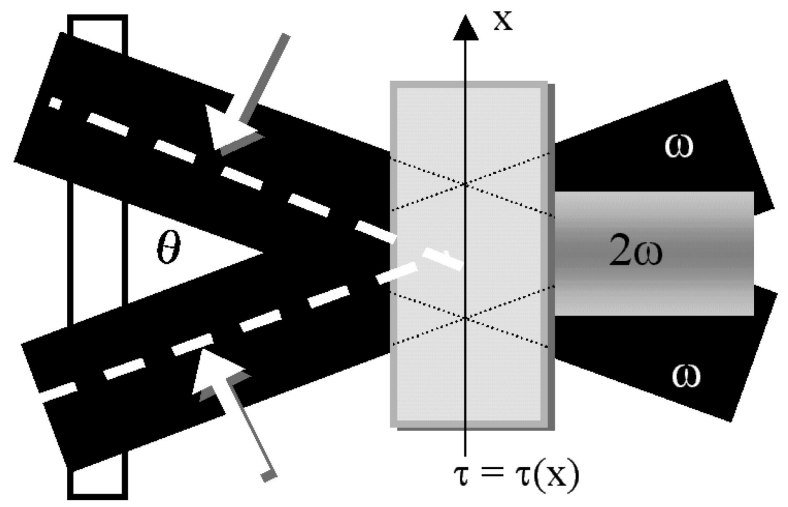

(b)

Fig. 1. Our experimental setup. (a) Top view: M1-M3, mirrors (M3 at the translation stage); BS, beam splitter; L, cylindrical lens; C, BBO crystal; W, webcam. (b) Side view.

changes that we propose are the reintroduction of the delay line eliminated by the Fresnel biprism, its replacement with a beam splitter and two simple mirrors, and the substitution of a simple webcam for a digital camera and an image grabber board. As a GRENOUILLE, we use a thick $\beta$-barium borate (BBO) crystal as a dispersive second-harmonic generation element. The idea is to have a reliable alternative method, uncomplicated and as cheap as possible, with which to explore the adjustability of the crossing angle and to evaluate the beam-profile superposition.

Some positive features that the GRENOUILLE method already has (and that are preserved in our setup) are listed next. First, it is possible to increase the phase-matching bandwidth by dithering the lateral position of the input beam. As was shown by O'Shea et al. ${ }^{12}$ it is possible to increase the range of angles in a given GRENOUILLE device, and thereby to increase the spectral range, without needing to make any changes to the device itself; this is necessary when one is handling more-complex or shorter pulses that are ultrabroadband. Another positive feature of this method is the possibility of measuring spatiotemporal distortion, or pulse-front tilt. ${ }^{13}$ Whereas GRENOUILLE traces are ordinarily centered on the zero of delay, a pulse with pulse-front tilt yields a trace whose center is shifted to a nonzero delay that is proportional to the pulse-front tilt. As a result, the trace-center shift reveals both the magnitude and the sign of the pulsefront tilt, independently of the temporal pulse intensity and phase. The effects of pulse-front tilt can then easily be removed from the trace, and the intensity and the phase versus time can also be retrieved, yielding a full description of the pulse in space and time. Finally, by use of the bootstrap method, a well-known statistical technique, it is possible to compute error bars automatically in ultrashort-pulse measurements. ${ }^{14}$ The bootstrap method allows us to characterize the uncertainty in the measured pulse intensity and phase in the presence of noise by generating a complete distribution of possible values for the desired parameters.

\section{Experiment}

As in the basic GRENOUILLE setup, we use a thick $(6 \times 5 \times 8 \mathrm{~mm}$, cut $28 \mathrm{deg})$ second-harmonic generation crystal (BBO) that performs the self-gating. Figure 1 shows the experimental setup that we have developed [Fig. 1(a), top view; Fig. 1(b), side view). Alternatively, in our setup we produce amplitude division in the original beam with a beam splitter and use two mirrors to superpose them. The beams cross in the crystal with a linear delay along the $X$ coordinate, given by the cross angle and the diameter of the beams. One of the mirrors and the beam splitter are fixed, so we need to align them only once. The other mirror can be used to generate additional delay between the pulses and also to change the crossing angle of the beams. The first impression caused by the reintroduction of the delay line in the setup is that we add only the complexity eliminated by the biprism, but the advantages prove to be manifold. For example, the angle mentioned above defines the delay range when the beams cross in the BBO crystal, which is fixed for the biprism, and therefore a connection among beam width, biprism apex angle, and crystal dimensions is established.

In the vertical direction no changes were introduced in the basic setup. The thick crystal has a relatively small phase-matching bandwidth for the sum frequency, so the phase-matched wavelength produced by it varies with angle. Along the $Y$ coordinate the beams are focused with a cylindrical lens $(f=100 \mathrm{~mm})$, so the convergence angle has enough aperture to accommodate all the phase-matched wavelengths that constitute the pulse. Thus the thick crystal also acts as a spectrometer. The groupvelocity mismatch (GVM) between fundamental and second harmonic accumulates dephasing along the crystal length $L$. Therefore, if $t_{p}$ is the pulse length, GVM $\times L \gg t_{p}$ is the condition with which to achieve the necessary spectral resolution. To prevent a pulse spread in time, the group-velocity dispersion (GVD) must also satisfy GVD $\times L \ll t_{c}$, where $t_{c}$ is the pulse 


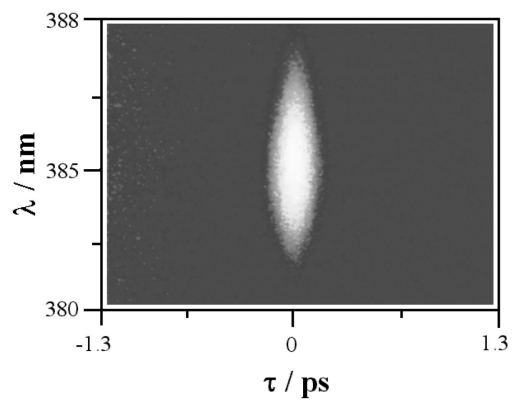

(a)

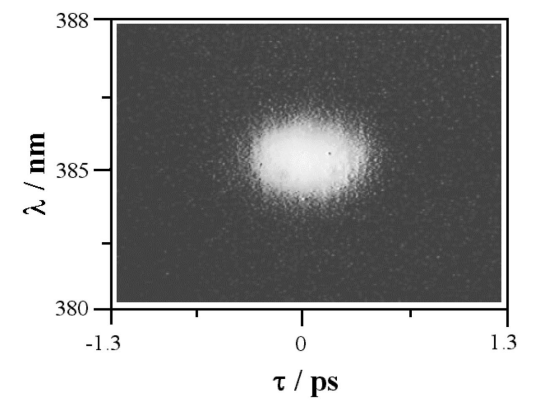

(b)

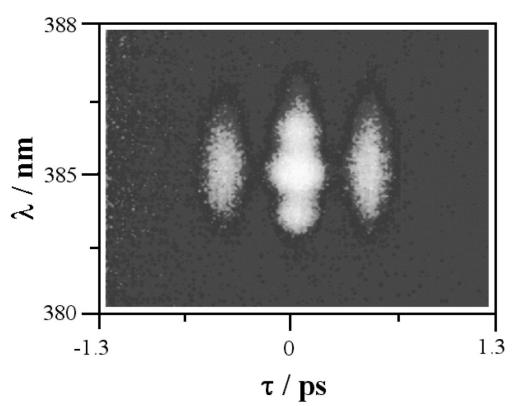

(c)

Fig. 2. Webcam images obtained with our setup (gray-scale intensity): (a) a transform-limited pulse; (b) chirped pulse; (c) result when a double pulse impinges on the GRENOUILLE.

coherence time. Together those relations are fulfilled if GVM/GVD $\gg t_{p} / t_{c}$, where the ratio is also determined by the time-bandwidth product of the pulse. GRENOUILLE ${ }^{11}$ can accurately measure a pulse with a time-bandwidth product (TBP) of as much as 10 (for a transform-limited pulse; TBP $=1$ ). To acquire the far-field image we use an unsophisticated webcam (288 $\times 352$ pixels), with an 8-bit analog-todigital converter (CMOS OmniVision OV7610), the same intensity resolution used in FROG traces. ${ }^{15}$

The femtosecond pulse source that we used to test our method was a commercial solid-state pumped (Verdi-5W) Ti:sapphire laser system (Coherent Mira 900 ), which supplied an average power of $200 \mathrm{~mW}$ of $180-\mathrm{fs}$ pulses at $800 \mathrm{~nm}$ with a repetition rate of $80 \mathrm{MHz}$. This laser delivers pulses that are Fouriertransform limited. Using them and some additional optics, we synthesized three types of pulse: Fouriertransform limited, chirped, and double pulse. To produce a chirped pulse we assembled a scheme with two prisms ${ }^{16}$ to produce the necessary dispersion. For the double pulse we used a Michelson interferometer to produce a variable delay between the two pulses generated by the division of the original pulse. The beam diameter at the GRENOUILLE entrance was $\sim 2.2 \mathrm{~mm}$. The webcam's sensitivity is inferior to that of a professional camera ${ }^{15}$ and therefore limits the minimum power required for taking a GRENOUILLE image to approximately $10 \mathrm{~mW}$, corresponding to pulses greater than $250 \mathrm{pJ}$ but that are usually available in femtosecond laser systems.

\section{Results and Discussion}

In Fig. 2 we present the webcam images obtained with our setup (gray-scale intensity). The first image, in Fig. 2(a), corresponds to a transform-limited pulse; that is, all the frequencies that made up the pulse are in phase. Figure 2(b) shows a chirped pulse. The second-order nonlinearity does not distinguish between positive and negative chirp ${ }^{17}$ because this nonlinearity is symmetric in time, so the figure is also symmetric about the time axis. Finally, Fig. 2(c) shows what happens when a double pulse impinges upon our GRENOUILLE setup. This image has three characteristic features, one at zero time delay and one each at the delays $-t$ and $+t$. Because the tem- poral shift generated by the Michelson interferometer corresponds to a linear phase in the frequency domain, the coherent superposition at zero delay gives fringes in the frequency direction.

These results are used as the input of the FrogGUI field envelope recovery program, ${ }^{18}$ and the electric field envelope profile that we wish to recover has the form $E(t)=E_{0}(t) \exp \left[i \omega_{0} t+i \phi(t)\right]$. The optical spectrum of each pulse is also measured independently by use of an optical multichannel analyzer with a $0.25-\mathrm{m}$ Jarrel-Ash spectrometer that has a $0.3-\mathrm{nm}$ resolution. This spectrum is used as a cross check for the quality of the GRENOUILLE recovered spectrum. First we recover the Fourier-transform-limited pulse. Figure 3(a) shows the recovered spectral intensity plotted with the experimentally measured spectrum. Excellent agreement can be observed in the region where the field amplitude is noticeable. Also shown is the spectral phase, which within the same range is a linear function of the wavelength, as should be expected for a Fourier-transform-limited pulse. Figure $3(\mathrm{~b})$ shows the time-dependent intensity and phase, corresponding to a hyperbolic secant function envelope of the electric field with a duration of $123 \mathrm{fs}$. Fig. 4 the chirped pulse is retrieved. The spectral and temporal phases of this pulse are quadratic functions of wavelength and time, respectively, as expected for such a pulse. Figure 5 shows the recovered double pulse and the characteristic frequency-domain fringes that resemble the two-slit interference pattern that is also marked by phase jumps.

The delay line introduced to regulate the beam overlap in our setup allowed further improvements to be made in the characterization of this kind of assembly. The original GRENOUILLE setup has a fixed time scan defined by biprism refractive index $(n)$ and angle $(\theta)$ through the split profile overlap, which, for a determined beam width $(w)$, occurs at a preestablished distance from the biprism ridge $[z=w / 2 \theta(n$ $-1)$. Another disadvantage of the biprism arrangement is the characteristic superposition of the split profile. The halved profiles imaged on the focus of the cylindrical lens do not permit a perfect wave-front match because of the asymmetric energy distribution perpendicular to the biprism ridge. The setup presented here introduces an overlap of mirror-imaged 


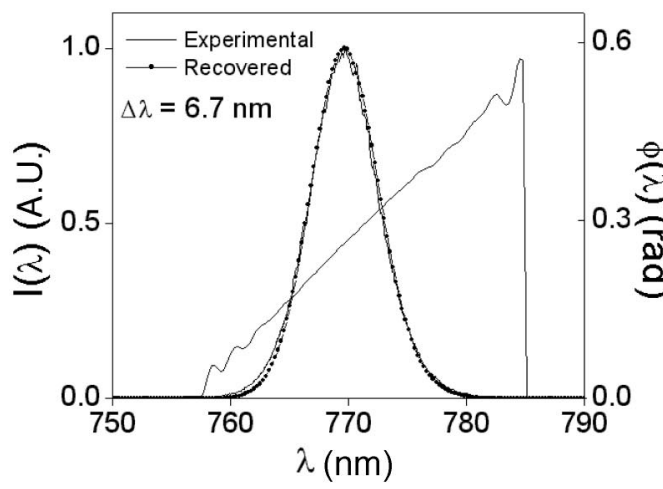

(a)

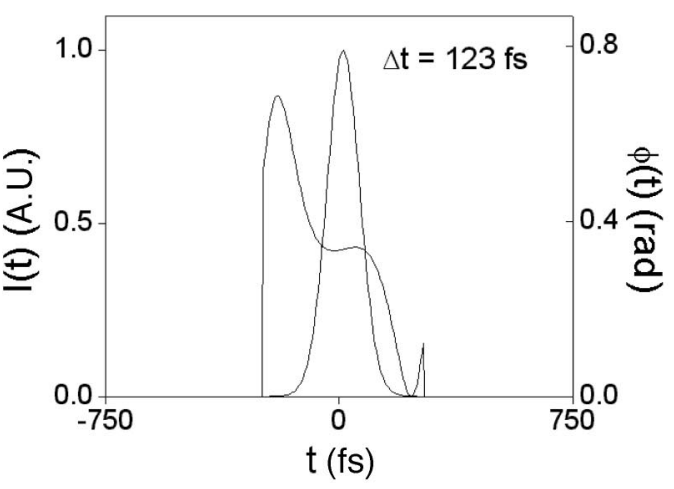

(b)

Fig. 3. (a) Spectral intensity recovered by the iterative program for the transform-limited pulse. The spectral phase is also shown, and the experimentally measured spectral intensity is juxtaposed. (b) Temporal intensity and temporal phase recovered by the iterative program. As can be seen, the phase is a linear function of time.

profiles, forcing a symmetric FROG picture even for a spatial chirp in the profile. The excellent superposition of a Gaussian amplitude profile in our setup improved the second-harmonic generation efficiency and, therefore, lowered the minimum power required for observation of the self-referenced image. A superposition of equally shaped profiles may also be devel- oped for the recognition of spatial chirp, in which an additional mirror is introduced for one of the beams.

The delay line offers in addition the possibility of analyzing the maximum value of the delay margin, i.e., the integral of the GRENOUILLE trace along the frequency axis. Figure 6 shows the result when the translation stage is varied, introducing a delay into

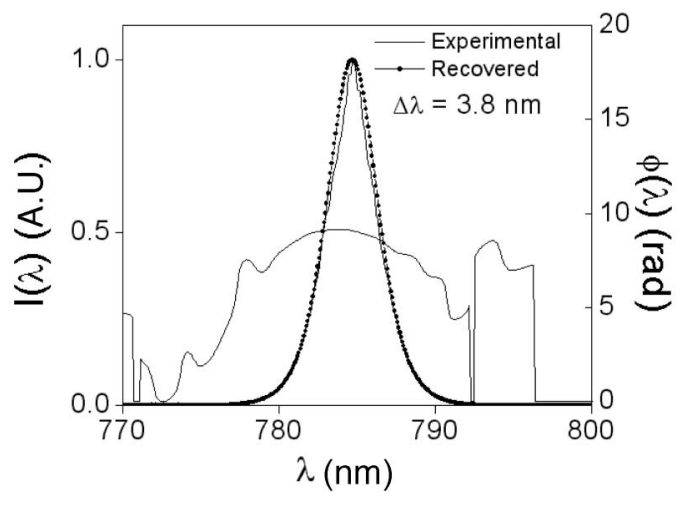

(a)

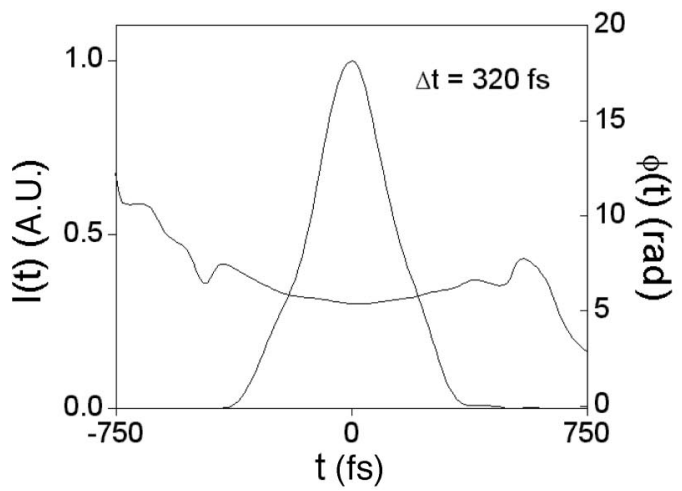

(b)

Fig. 4. (a) Same as Fig. 3(a) but for the chirped pulse. (b) Same as Fig. 3(b) but for the chirped pulse. As can be seen, the phase is a quadratic function of time.

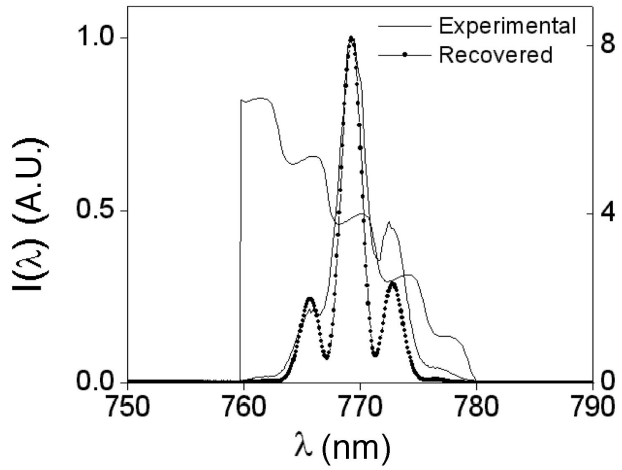

(a)

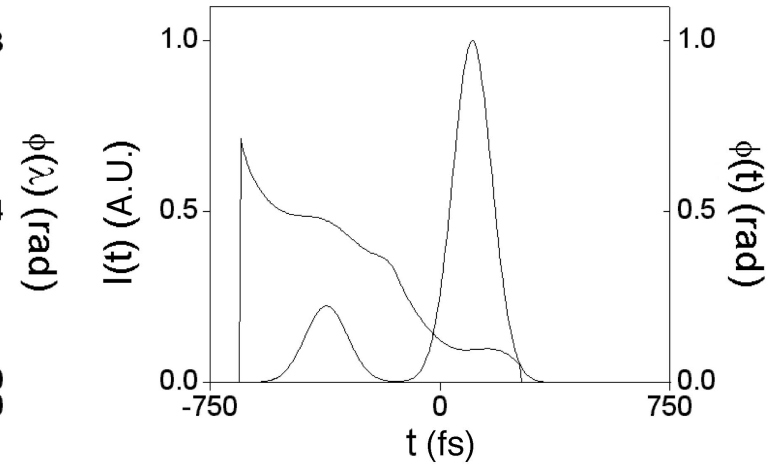

(b)

Fig. 5. (a) Same as Fig. 3(a) but for the double pulse. (b) Same as Fig. 3(b) but for the double pulse. 


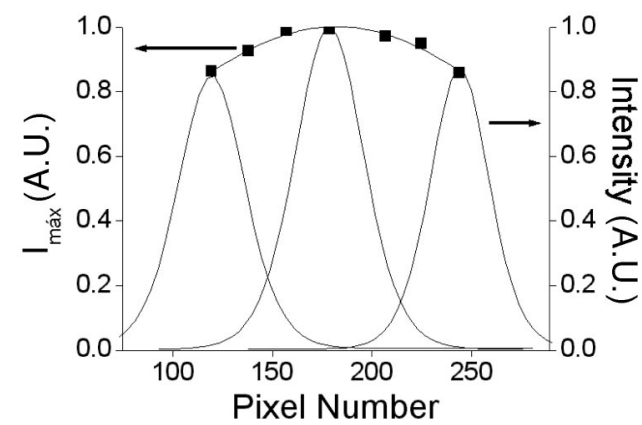

Fig. 6. Variation of the maximum value of the delay margin (filled squares). Also shown are three curves obtained for three different delay positions of the translation stage.

one of the arms. The maximum intensity of the delay margin is not constant but reflects the Gaussian intensity of the spatial beam profile. As can be seen, the maximum variation is less than $20 \%$ within the $1500-f$ s observable range. For relatively short pulses $(\sim 300 \mathrm{fs})$, the error introduced by this effect is minor. But for double or multiple pulses this additional effect must be regarded as well. A proper normalization must be made that takes into account the observed variation and includes the time-scan limit imposed by the crystal's dimensions. In the original GRENOUILLE setup the analysis described above cannot be made. Also, the diffraction generated in the biprism apex region causes the borders of the temporal scan region to deteriorate, thus restricting the effective second-harmonic generation, as reported by Akturk et al. ${ }^{19}$ especially if long structured pulses are evaluated. This effect does not occur in our setup. The only drawback, as expected, is the additional alignment requirement, but this alignment is simple and need be made only once for the chosen crossing angle; thus the original advantage of GRENOUILLE, that of single-shot characterization, is retained.

\section{Concluding Remarks}

Our version of GRENOUILLE has all characteristics of the original scheme and some new noteworthy features; it constitutes an alternative method for those who do not have a Fresnel biprism with a designed apex angle at hand. We used the additional degree of freedom that the translating mirror introduces to evaluate the beam-profile convolution and also to change the time-scan width. Another feature of this method is that we use reflective optics almost exclusively, minimizing the GVD that occurs when the beam passes through refractive materials. The webcam imaging exhibited no artifacts because the beam exposure was controlled to prevent saturation. Simulated theoretical pulses with the given wavelength bandwidth of our laser setup produced temporal widths that are in agreement with our experimental results. Also, this method shows the correct expected phase evolution of the respective pulses. So the proposed modification of the GRENOUILLE method works well and can easily be used on a daily basis for pulse characterization.
The authors are thankful for the partial support of the Programa Brasileiro de Apoio ao Desenvolvimento Cientifico e Tecnológico III and the Conselho Nacional de Desenvolvimento Cientifico e Tecnológico agency.

\section{References and Notes}

1. H. Kawashima, M. M. Wefers, and K. A. Nelson, "Femtosecond pulse shaping, multiple-pulse spectroscopy, and optical control," Annu. Rev. Phys. Chem. 46, 627-656 (1995).

2. A. M. Weiner, D. E. Leaird, G. P. Wiederrecht, and K. A. Nelson, "Femtosecond pulse sequences used for optical manipulation of molecular-motion," Science 247, 1317-1319 (1990).

3. N. Dudovich, B. Dayan, S. M. G. Faeder, and Y. Silberberg, "Transform-limited pulses are not optimal for resonant multiphoton transitions," Phys. Rev. Lett. 86, 47-50 (2001).

4. D. Meshulach and Y. Silberberg, "Coherent quantum control of two-photon transitions by a femtosecond laser pulse," Nature 396, 239-242 (1998).

5. R. Trebino and D. J. Kane, "Using phase retrieval to measure the intensity and phase of ultrashort pulses: frequencyresolved optical gating," J. Opt. Soc. Am. A 10, 1101-1111 (1993).

6. R. Trebino, K. W. DeLong, D. N. Fittinghoff, J. N. Sweetser, M. A. Krumbügel, B. A. Richman, and D. J. Kane, "Measuring ultrashort laser pulses in the time-frequency domain using frequency-resolved optical gating," Rev. Sci. Instrum. 68, 3277-3295 (1997).

7. C. Iaconis and I. A. Walmsley, "Self-referencing spectral interferometry for measuring ultrashort optical pulses," IEEE J. Quantum Electron. 35, 501-509 (1999).

8. L. Gallmann, D. H. Sutter, N. Matuschek, G. Steinmeyer, U. Keller, C. Iaconis, and I. A. Walmsley, "Characterization of sub-6-fs optical pulses with spectral phase interferometry for direct electric-field reconstruction," Opt. Lett. 24, 1314-1316 (1999).

9. C. Dorrer and I. A. Walmsley, "Accuracy criterion for ultrashort pulse characterization techniques: application to spectral phase interferometry for direct electric field reconstruction," J. Opt. Soc. Am. B 19, 1019-1029 (2002).

10. C. Dorrer and I. A. Walmsley, "Precision and consistency criteria in spectral phase interferometry for direct electric-field reconstruction," J. Opt. Soc. Am. B 19, 1030-1038 (2002).

11. P. O'Shea, M. Kimmel, X. Gu, and R. Trebino, "Highly simplified device for ultrashort-pulse measurement," Opt. Lett. 26, 932-934 (2001).

12. P. O'Shea, M. Kimmel, and R. Trebino, "Increased phasematching bandwidth in simple ultrashort-laser-pulse measurements,” J. Opt. B Quantum Semiclass. Opt. 4, 44-48 (2002).

13. S. Akturk, M. Kimmel, P. O'Shea, and R. Trebino, "Measuring pulse-front tilt in ultrashort pulses using GRENOUILLE," Opt. Express 11, 491-501 (2003), http://www.opticsexpress.org.

14. Z. Wang, E. Zeek, R. Trebino, and P. Kvam, "Beyond error bars: understanding uncertainty in ultrashort-pulse frequencyresolved-optical-gating measurements in the presence of ambiguity," Opt. Express 11, 3518-3527 (2003), http://www. opticsexpress.org.

15. K. W. Delong, D. N. Fittinghoff, and R. Trebino, "Practical issues in ultrashort-laser-pulse measurement using frequencyresolved optical gating," IEEE J. Quantum Electron. 32, 12531264 (1996).

16. R. L. Fork, O. E. Marinez, and J. P. Gordon, "Negative dispersion using pairs of prisms," Opt. Lett. 9, 150-152 (1984). 
17. K. W. DeLong, R. Trebino, J. Hunter, and W. E. White, "Frequency-resolved optical gating with the use of secondharmonic generation," J. Opt. Soc. Am. B 11, 2206-2215 (1994).

18. Phase retrieval for Frequency-Resolved Optical Gating using
Genetic Algorithms and Generalized Projections. Copyright 1998 Jeff Nicholson, jwn@ofsoptics.com.

19. S. Akturk, M. Kimmel, P. O'Shea, and R. Trebino, "Extremely simple device for measuring 20-fs pulses," Opt. Lett. 29, 10251027 (2004). 